\title{
A STUDY OF ANALYTIC SURFACES BY MEANS OF A PROJECTIVE THEORY OF ENVELOPES $\left({ }^{1}\right)$
}

\author{
BY \\ E. GREER AND P. O. BELL
}

1. Introduction. Let $Q_{x}$ denote a quadric associated with a generic point $x$ of an analytic surface $S$, and let $C_{\lambda}$ denote a curve of $S$ passing through $x$. Let $X$ denote a point of $C_{\lambda}$ in the neighborhood of $x$. The limit of the curve of intersection of the quadrics $Q_{x}$ and $Q_{X}$ as $X$ approaches $x$ is called the characteristic curve of $Q_{x}$ with respect to the curve $C_{\lambda}$ at $x$. The quadric cone which contains this characteristic curve and has its vertex at $x$ will be called the characteristic cone $\mathcal{C}_{\lambda}$ of $Q_{x}$ at $x$.

This paper applies a projective theory of envelopes [2] $\left(^{2}\right)$ to determine and study the cones $C_{\lambda}$ associated with significant families of quadrics having contact of the second order with $S$ at $x$.

The geometric inter-relations of the cone $\mathcal{C}_{\lambda}$, the quadric $Q_{x}$, and the curve $C_{\lambda}$ are investigated. The principal results are geometric characterizations of significant families of quadrics, systems of hypergeodesics, the canonical pencil, projective curvatures of a curve $C_{\lambda}$, the general transformation of Cech, the curves of Darboux and Segre, and a class of generalized pangeodesics. Among the quadrics characterized are the Moutard pencil of quadrics, the Davis quadrics, and the asymptotic osculating quadrics of Bompiani. These quadrics have been defined, heretofore, by distinct and apparently unrelated properties. They are here shown to be members of a system of quadrics characterized by a special property of their characteristic cones. Other properties serve to characterize an invariant pencil of Darboux quadrics.

2. Preliminaries. Let an analytic non-ruled surface $S$ be referred to its asymptotic net as parametric, and let the projective homogeneous coordinates of a general point $x$ of $S$ be normalized so that they satisfy the Fubini canonical system of differential equations

$$
\begin{aligned}
& x_{u u}=p x+\theta_{u} x_{u}+\beta x_{v}, \\
& x_{v v}=q x+\gamma x_{u}+\theta_{v} x_{v}, \quad \theta=\log \beta \gamma .
\end{aligned}
$$

Since the points $x, x_{u}, x_{v}, x_{u v}$ are linearly independent, the general homogeneous coordinates of any point $X$ may be written in the form

$$
X=x^{0} x+x^{1} x_{u}+x^{2} x_{v}+x^{3} x_{u v}
$$

Presented to the Society, November 29, 1946; received by the editors June 13, 1947.

(1) This paper is, except for minor changes, E. Greer's University of Kansas doctoral dissertation, which was prepared under the supervision of P. O. Bell.

(2) Numbers in brackets refer to the references cited at the end of the paper. 
The local coordinates of $X$ with respect to the tetrahedron $\left(x, x_{u}, x_{v}, x_{u v}\right)$ are proportional to $x^{0}, x^{1}, x^{2}, x^{3}$ if the unit point of the reference frame is suitably chosen. Referred to this coordinate system the three-parameter family of quadric surfaces having contact of the second order with $S$ at $x$ has the equation

$$
x^{1} x^{2}-x^{0} x^{3}+k_{1} x^{1} x^{3}+k_{2} x^{2} x^{3}+k_{3}\left(x^{3}\right)^{2}=0,
$$

where $k_{1}, k_{2}$, and $k_{3}$ are arbitrary functions of $u, v$. If, and only if, $k_{1}=k_{2}=0$, the quadrics (2.2) are quadrics of Darboux defined by

$$
x^{1} x^{2}-x^{0} x^{3}+k_{3}\left(x^{3}\right)^{2}=0 .
$$

3. The characteristic cone. Conjugate and triple point tangents. A projective theory of envelopes is based on the use of the formulas for differentiation of local point coordinates. These formulas are [2, pp. 27-28]

$$
\partial x^{i} / \partial u^{\alpha}=-x^{h} \Gamma_{h \alpha}^{i}, \quad i, h=0,1,2,3, \quad \alpha=1,2, \quad u^{1}=u, \quad u^{2}=v,
$$

in which the repeated index $h$ denotes summation through the indicated range, and the functions $\Gamma_{h \alpha}^{i}$ are (in the case of the present coordinate system) related to the coefficients of $(2.1)$ by the equations

$$
\begin{array}{lll}
\Gamma_{0 \alpha}^{i}=\delta_{\alpha}^{i}, & \Gamma_{\alpha \epsilon}^{i}=\Gamma_{\epsilon \alpha}^{i}=\delta_{3}^{i}, & \alpha \neq \epsilon, \quad \epsilon, \alpha=1,2, \\
\Gamma_{11}^{0}=p, & \Gamma_{\alpha \alpha}^{\alpha}=\Gamma_{3 \alpha}^{3}=\frac{\partial \theta}{\partial u^{\alpha}}, \quad \Gamma_{\alpha \alpha}^{3}=0, \quad \Gamma_{22}^{0}=q, \\
\Gamma_{22}^{1}=\gamma, & \Gamma_{11}^{2}=\beta, \quad \Gamma_{3 \alpha}^{\alpha}=\kappa, \quad \Gamma_{32}^{1}=\chi, \\
\Gamma_{31}^{2}=\pi, & \Gamma_{31}^{0}=p_{v}+\beta q, \quad \Gamma_{32}^{0}=q_{u}+\gamma p, \\
\kappa=\beta \gamma+\theta_{u v}, & \pi=p+\beta_{v}+\beta \theta_{v}, & \chi=q+\gamma_{u}+\gamma \theta_{u},
\end{array}
$$

in which the deltas denote the Kronecker symbols.

Let $C_{\lambda}$ denote a curve of $S$ defined by the curvilinear differential equation $d v-\lambda(u, v) d u=0$. Let equation (2.2) be represented by $f=0$. The equations of the characteristic curve of (2.2) are therefore given by

$$
f=0, \quad f_{u}+\lambda f_{v}=0,
$$

in which the right members of (3.1) are substituted for the partial derivatives of local point coordinates. The second equation of (3.2) is found to be

$$
\begin{aligned}
& \left(\beta+k_{1} \lambda\right)\left(x^{1}\right)^{2}+\left(\theta_{u}+\lambda \theta_{v}+k_{1}+k_{2} \lambda\right) x^{1} x^{2}+\left(\gamma \lambda+k_{2}\right)\left(x^{2}\right)^{2} \\
& \quad+\left(k_{1}+k_{2} \lambda-\theta_{u}-\lambda \theta_{v}\right) x^{0} x^{3}+\left(\pi+\kappa \lambda+k_{1} \theta_{u}+k_{1} \lambda \theta_{v}+k_{1} \theta_{u}\right. \\
& \left.\quad+k_{2} \beta-p-k_{1}^{\prime}+2 k_{3} \lambda\right) x^{1} x^{3}+\left(\kappa+\chi \lambda+k_{2} \theta_{u}+k_{2} \lambda \theta_{v}\right. \\
& \left.\quad+k_{1} \gamma \lambda+k_{2} \theta_{v} \lambda-q \lambda-k_{2}^{\prime}+2 k_{3}\right) x^{2} x^{3}+\left(k_{1} \kappa+k_{1} \lambda \chi+k_{2} \pi\right. \\
& \left.\quad+k_{2} \lambda \kappa-p_{v}-\beta q-q_{u} \lambda-\gamma p \lambda-k_{3}^{\prime}+2 k_{3} \theta_{u}+2 k_{3} \lambda \theta_{v}\right)\left(x^{3}\right)^{2}=0
\end{aligned}
$$


in which accents indicate differentiation with respect to $u$.

The equation of the characteristic cone $C_{\lambda}$ is found, by eliminating $x^{0} x^{3}$ between equations (2.2) and (3.3), to be

$$
\begin{aligned}
(\beta+ & \left.k_{1} \lambda\right)\left(x^{1}\right)^{2}+2\left(k_{1}+k_{2} \lambda\right) x^{1} x^{2}+\left(\gamma \lambda+k_{2}\right)\left(x^{2}\right)^{2} \\
& +\left(k_{1}^{2}+k_{1} k_{2} \lambda+\theta_{u} k_{1}+\beta k_{2}-k_{1}^{\prime}+\beta \psi+\kappa \lambda+2 k_{3} \lambda\right) x^{1} x^{3} \\
& +\left(k_{2}^{2} \lambda+k_{1} k_{2}+k_{2} \theta_{v} \lambda+k_{1} \gamma \lambda-k_{2}^{\prime}+\gamma \phi \lambda+\kappa+2 k_{3}\right) x^{2} x^{3} \\
& +\left[\left(k_{1}+k_{2} \lambda\right)\left(\kappa+k_{3}\right)+\pi k_{2}+\chi k_{1} \lambda-p_{v}-\beta q-q_{u} \lambda-\gamma p \lambda\right. \\
& \left.+\theta^{\prime} k_{3}-k_{3}^{\prime}\right]\left(x^{3}\right)^{2}=0,
\end{aligned}
$$

where $\psi=\left(\log \beta^{2} \gamma\right)_{v}, \phi=\left(\log \beta \gamma^{2}\right)_{u}$.

Let $t_{\tau}$ denote the tangent line to $S$ at $x$ whose direction is $\tau$. Since the vertex of the cone $\mathcal{C}_{\lambda}$ is the point $x$, the polar plane of a point $P$ of $t_{\tau}$ with respect to the cone $\mathcal{C}_{\lambda}$ is independent of the choice of $P$ on $t_{\tau}$. The equation of this polar plane is

$$
\begin{aligned}
2[\beta & \left.+(\lambda+\tau) k_{1}+\lambda \tau k_{2}\right] x^{1}+2\left[\gamma \lambda \tau+k_{1}+(\lambda+\tau) k_{2}\right] x^{2} \\
& +\left[\left(k_{1}^{2}+\theta_{u} k_{1}+\beta k_{2}-k_{1}^{\prime}+\beta \psi\right)+(\lambda+\tau)\left(k_{1} k_{2}+\kappa+2 k_{3}\right)\right. \\
& \left.+\left(\lambda k_{2}^{2}+\theta_{v} \lambda k_{2}+\gamma \lambda k_{1}-k_{2}^{\prime}+\gamma \lambda \phi\right) \tau\right] x^{3}=0 .
\end{aligned}
$$

Let $\pi_{x}$ denote the tangent plane of $S$ at $x$. The intersection of (3.5) and $\pi_{x}$ is in the direction defined by

$$
x^{2} / x^{1}=-\left[\beta+(\lambda+\tau) k_{1}+\lambda \tau k_{2}\right] /\left[\gamma \lambda \tau+(\lambda+\tau) k_{2}+k_{1}\right] .
$$

The equations of the lines of intersection of the cone $\mathcal{C}_{\lambda}$ with $\pi_{x}$ are (3.7) $x^{3}=0,\left(\beta+k_{1} \lambda\right)\left(x^{1}\right)^{2}+2\left(k_{1}+k_{2} \lambda\right) x^{1} x^{2}+\left(\gamma \lambda+k_{2}\right)\left(x^{2}\right)^{2}=0$.

The directions of these lines are conjugate directions $\pm \eta$ if, and only if, $k_{1}+\lambda k_{2}=0$, where

$$
\eta=\left[-\left(\beta+k_{1} \lambda\right) /\left(\gamma \lambda+k_{2}\right)\right]^{1 / 2} .
$$

It follows that if $k_{1}=k_{2}=0$,

$$
\lambda=-\beta / \gamma \eta^{2} .
$$

The characteristic cone $\mathcal{C}_{\lambda}$ determined by a quadric of Darboux will be denoted by $\mathcal{C}_{\lambda, D}$.

THEOREM 3.1. The directions of the lines of intersection of a characteristic cone $C_{\lambda, D}$ with the plane $\pi_{x}$ are conjugate directions $\pm \eta$. The direction $\lambda$ is the $R_{\eta}$-correspondent of $\eta[1$, p. 392].

If $\lambda$ coincides with one of the directions of the lines (3.7), $\lambda$ is found to be a solution of the equation

$$
\beta+3 k_{1} \lambda+3 k_{2} \lambda^{2}+\gamma \lambda^{3}=0
$$


for the directions of the triple point tangents of the curve of intersection of $S$ and the quadric (2.2).

TheOREM 3.2. The cone $C_{\lambda}$ intersects $\pi_{x}$ in the tangent to the curve $C_{\lambda}$ if, and only if, $t_{\lambda}$ is one of the triple point tangents of the curve of intersection of the surface $S$ with the quadric (2.2).

Let $N_{\lambda}$ denote the conjugate net of $S$ whose tangents at $x$ are in the directions $\pm \lambda$. On making use of the condition, $k_{1}+\lambda k_{2}=0$, that the cone $C_{\lambda}$ intersect $\pi_{x}$ in conjugate tangents together with the condition (3.8), the following theorem results.

TheOREM 3.3. The cone $\mathcal{C}_{\lambda}$ intersects the plane $\pi_{x}$ in the tangents at $x$ to the conjugate net $N_{\lambda}$ if, and only if, $\lambda$ is a direction of Darboux.

4. Condition that $C_{\lambda, D}$ reduce to two planes. Quadric of Lie. The condition that $C_{\lambda, D}$ reduce to two planes is readily found by equating to zero $k_{1}, k_{2}$, and the discriminant of (3.4) to be

$$
\begin{aligned}
& \beta^{-1}\left(2 k_{3}+\kappa\right)^{2} \lambda^{3}+\left(4 k_{3_{v}}-4 \theta_{v} k_{3}+4 q_{u}+4 \gamma p+2 \psi \kappa\right. \\
& \left.\quad+4 \psi k_{3}+\gamma \phi^{2}\right) \lambda^{2}+\left(4 k_{3_{u}}-4 \theta_{u} k_{3}+4 p_{v}+4 \beta q+2 \phi \kappa\right. \\
& \left.\quad+4 \phi k_{3}+\beta \psi^{2}\right) \lambda+\gamma^{-1}\left(2 k_{3}+\kappa\right)^{2}=0 .
\end{aligned}
$$

THEOREM 4.1. The cone $C_{\lambda, D}$ reduces to two intersecting planes for each of three directions. Two of these directions are the asymptotic directions if, and only if, the quadric is the quadric of Lie $\left(k_{3}=-\kappa / 2\right)$; the third direction is then defined by

$$
\lambda=-\frac{8 p_{v}+2(\beta \psi)_{v}-2 \beta \gamma \phi+4 \beta q+\beta \psi^{2}}{8 q_{u}+2(\gamma \phi)_{u}-2 \beta \gamma \psi+4 \gamma p+\gamma \phi^{2}} .
$$

5. Polar planes with respect to $C_{\lambda, D}$. Let $p_{\mu, \lambda}$ denote the polar plane of an arbitrary point on $t_{\mu}$ with respect to the cone $C_{\lambda, D}$. The equation of the plane $p_{\mu, \lambda}$ is found to be

$$
2 \beta x^{1}+2 \gamma \lambda \mu x^{2}+\left[\beta \psi+\gamma \phi \lambda \mu+(\lambda+\mu)\left(\kappa+2 k_{3}\right)\right] x^{3}=0 .
$$

This plane intersects $\pi_{x}$ in a line whose direction coincides with $\lambda$ if $\mu$ is defined by

$$
\mu=-\beta / \gamma \lambda^{2} .
$$

THEOREM 5.1. The polar plane $p_{\mu, \lambda}$ passes through the tangent $t_{\lambda}$ of $S$ at $x$ if and only if $t_{\mu}$ is the $R_{\lambda}$-correspondent of $t_{\lambda}$. The polar plane $p_{\lambda, \lambda}$ passes through the $R_{\lambda}$-correspondent of $t_{\lambda}$.

The equation of an arbitrary plane through $t_{\lambda}$ is of the form

$$
2 \lambda^{2} x^{1}-2 \lambda x^{2}+\rho x^{3}=0 .
$$


The equation of the polar plane $p_{h \lambda, \lambda}, h=$ const., may be found at once from (5.1) to be

$$
\beta\left(2 x^{1}+\psi x^{3}\right)+(1+h)\left(\kappa+2 k_{3}\right) \lambda x^{3}+\gamma\left(2 x^{2}+\phi x^{3}\right) h \lambda^{2}=0 .
$$

If $(1+h)\left(\kappa+2 k_{3}\right)=0$, this plane generates a pencil as $\lambda$ varies, whose axis is defined by the equations

$$
2 x^{1}+\psi x^{3}=0, \quad 2 x^{2}+\phi x^{3}=0 .
$$

THEOREM 5.2. The polar plane $p_{-\lambda, \lambda}$ is independent of the choice of the quadric of Darboux and for each value of $\lambda$ passes through the directrix $d^{\prime}$ of Wilczynski. The polar plane $p_{h \lambda, \lambda}, h \neq-1$, for each direction $\lambda$ passes through the directrix of Wilczynski if, and only if, the quadric of Darboux is the quadric of Lie.

To find the equations of the planes through $t_{\lambda}$ which are tangent to the cone $\mathcal{C}_{\lambda, D}$, equate $k_{1}$ and $k_{2}$ to zero in (3.4), eliminate $x^{3}$ between the resulting equations (5.3) and (3.4), and set the discriminant of this eliminant equal to zero. This discriminant equation is found to be of the form

$$
\beta \gamma \rho^{2}+2\left[\beta \gamma \lambda(\phi-\psi \lambda)+\left(\beta-\gamma \lambda^{3}\right)\left(\kappa+2 k_{3}\right)\right] \rho+(1)=0,
$$

in which the terms denoted by (1) are immaterial in the present considerations. The roots of this quadratic equation in $\rho$ are the values of $\rho$ for which the plane (5.3) is tangent to $\mathcal{C}_{\lambda, D}$. The harmonic conjugate of $\pi_{x}$ with respect to the two tangent planes to $C_{\lambda, D}$ through $t_{\lambda}$, therefore, has the equation (5.3) where $\rho$ is one-half the sum of the roots of (5.4), that is

$$
\rho=\psi \lambda^{2}-\phi \lambda+\left(\gamma \lambda^{3}-\beta\right)\left(\kappa+2 k_{3}\right) / \beta \gamma .
$$

This plane is identical with the polar plane $p_{\mu, \lambda}$ where $\mu$ is defined by (5.2). The following theorem incorporates the result just proved with others which may be readily derived.

THEOREM 5.3. The polar plane $p_{\mu, \lambda}\left(p_{\lambda, \lambda}\right)$, where $t_{\mu}$ is the $R_{\lambda}$-correspondent of $t_{\lambda}$, is the harmonic conjugate of $\pi_{x}$ with respect to the two tangent planes to $\mathcal{C}_{\lambda, D}$ through $t_{\lambda}\left(t_{\mu}\right)$. The plane $p_{\mu, \lambda}$, for each direction $\lambda$, passes through the directrix $d^{\prime}$ of Wilczynski if, and only if, the quadric of Darboux is the quadric of Lie.

The osculating plane of the curve $C_{\lambda}$ at $x$ has for its equation (5.3) where $\rho$ is defined by

$$
\rho=\lambda^{\prime}+\beta-\theta_{u} \lambda+\theta_{v} \lambda^{2}-\gamma \lambda^{3} .
$$

This plane coincides with the plane $p_{\mu, \lambda}$ where $\mu$ is defined by (5.2) if, and only if, the values for $\rho$ defined by (5.5) and (5.6) are identical. This condition demands that $C_{\lambda}$ be a curve of a family of hypergeodesics defined by the equation

$$
\lambda^{\prime}=-\eta \beta-(\log \gamma)_{u} \lambda+(\log \beta)_{v} \lambda^{2}+\eta \gamma \lambda^{3},
$$


where $\eta=\left(2 \beta \gamma+\theta_{u v}+2 k_{3}\right) / \beta \gamma$. A distinct family of hypergeodesics is thus associated with each quadric of Darboux. The cusp-axis of any family of these hypergodesics is the directrix $d^{\prime}$ of Wilczynski.

Because of the relationships of these hypergeodesics to the quadrics of Darboux and the directrix of Wilczynski it seems appropriate to call them the $W_{D \text {-geodesics. }}$

THEOREM 5.4. The polar plane $p_{\mu, \lambda}$ where $t_{\mu}$ is the $R_{\lambda}$-correspondent of $t_{\lambda}$ coincides with the osculating plane of $C_{\lambda}$ at $x$ if, and only if, the curve $C_{\lambda}$ is a $W_{D}$-geodesic defined by (5.7). If $\eta$ has the value 1 , the $W_{D-\text { geodesics are the }}$ union curves of the congruence $\Gamma^{\prime}$ of the directrices of Wilczynski.

Another system of $W_{D}$-geodesics may be characterized in association with each quadric of Darboux by replacing in the above characterization the plane $p_{\mu, \lambda}$ by the plane $p_{-\mu,-\lambda}$. The equation of this system is again of the form (5.7), but with $\eta$ defined by the relation $\eta=-\left(\theta_{u v}+2 k_{3}\right) / \beta \gamma$. This system of $W_{D^{-}}$geodesics defined with respect to a quadric can coincide with the first system defined for the same quadric if, and only if, the quadric is the quadric of Lie. In this case the $W_{D^{-}}$geodesics are the union curves of the congruence $\Gamma^{\prime}$ of directrices of Wilzcynski. If $\eta=-1$, the second system of $W_{D}$-geodesics consists of the dual union curves of the congruence $\Gamma$ of directrices of Wilczynski.

6. $W_{D}$-geodesic curvature and the Darboux pencil. Let $\pi_{0}$ denote the osculating plane of the curve $C_{\lambda}$ at $x$, let $\pi_{1}$ denote the plane containing the tangent $t_{\lambda}$ and the cusp-axis $[5$, p. 190] of the pencil of conjugate nets defined by

$$
d v^{2}-\lambda^{2} h^{2} d u^{2}=0, \quad h=\text { const., }
$$

and let $\pi_{2}$ denote the plane $p_{\mu, \lambda}$ where $\mu$ is defined by (5.2). The equations of $\pi_{0}, \pi_{2}$, and $\pi_{1}$ are of the form (5.3) where the corresponding functions $\rho$ are given by (5.6), (5.5), and $\rho=\lambda^{\prime}-\theta_{u} \lambda+\theta_{v} \lambda^{2}$, respectively. The $W_{D}$-geodesic curvature of the curve $C_{\lambda}$ at $x$ will be defined as the cross ratio of the planes $\pi_{x}, \pi_{0}, \pi_{1}, \pi_{2}$ and will be denoted by $\sigma_{1}$, which is found to be given by

$$
\begin{aligned}
\sigma_{1} & =\left(\pi_{x}, \pi_{0}, \pi_{1}, \pi_{2}\right) \\
& =\left[\lambda^{\prime}+\gamma^{-1} \eta+(\log \gamma)_{u} \lambda-(\log \beta)_{v} \lambda^{2}-\beta^{-1} \eta \lambda^{3}\right] /\left(\beta-\gamma \lambda^{3}\right)
\end{aligned}
$$

where $\eta=\theta_{u v}+2 \beta \gamma+2 k_{3}$. Hence

$$
\begin{aligned}
\lambda^{\prime}= & -\gamma^{-1}\left[\theta_{u v}+\left(2-\sigma_{1}\right) \beta \gamma+2 k_{3}\right]-(\log \gamma)_{u} \lambda \\
& +(\log \beta)_{v} \lambda^{2}+\beta^{-1}\left[\theta_{u v}+\left(2-\sigma_{1}\right) \beta \gamma+2 k_{3}\right] \lambda^{3} .
\end{aligned}
$$

This equation defines a system of hypergeodesics. A comparison of the equations (5.7) and (6.1) reveals that a curve whose $W_{D \text {-geodesic curvature vanishes }}$ identically is a $W_{D}$-geodesic of the system (5.7).

The hypergeodesics (6.1) are the union curves of the congruence of the directrices of Wilczynski if, and only if, 


$$
k_{3}=-\left[\left(1-\sigma_{1}\right) \beta \gamma+\theta_{u v}\right] / 2 .
$$

This condition yields the result that any quadric of Darboux can be char-

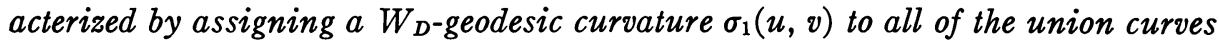
of the congruence of the directrices of Wilczynski. In particular if a constant $h$ is the value assigned to $\sigma_{1}$, the equation of the resulting quadric of Darboux is

$$
2\left(x^{1} x^{2}-x^{0} x^{3}\right)-\left[(1-h) \beta \gamma+\theta_{u v}\right]\left(x^{3}\right)^{2}=0 .
$$

This is the equation of a well known invariant pencil of Darboux quadrics. The form of equation (6.1) is such that the following theorem may be easily proved.

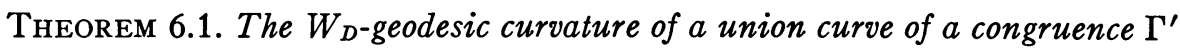
at $x$ is independent of the direction of the curve at $x$ if, and only if, $\Gamma^{\prime}$ is the congruence of the directrices $d^{\prime}$ of Wilczynski and $k_{3}$ is defined by (6.2). This curvature is constant along the curve $C_{\lambda}$ if the quadric is a member of the Darboux invariant pencil (6.3). The values 0,1 , and $1 / 3$ for $\sigma_{1}$ correspond to the quadrics of Lie, Wilczynski, and Fubini, respectively.

The form of the right member of the equation which defines $\sigma_{1}$ reveals that

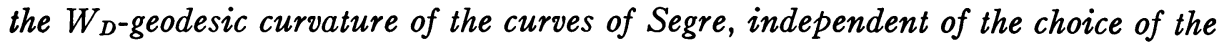
quadric of Darboux, is undefined.

7. Pangeodesic curvature and the Darboux pencil. The characteristic curve, of the quadric (2.2), which corresponds to the direction $\lambda$ at $x$ includes both asymptotic tangents at $x$ if, and only if, the coefficients of $\left(x^{1}\right)^{2}$ and $\left(x^{2}\right)^{2}$ in equation (3.3) vanish; that is, equation (2.2) becomes

$$
x^{1} x^{2}-x^{0} x^{3}-\beta \lambda^{-1} x^{1} x^{3}-\gamma \lambda x^{2} x^{3}+k_{3}\left(x^{3}\right)^{2}=0 .
$$

For the quadric (7.1) equation (3.6) reduces to

$$
x^{2} / x^{1}=-\tau \text {. }
$$

This is the direction conjugate to $\tau$.

Let $\pi_{-\lambda}$ denote the polar plane of an arbitrary point on $t_{-\lambda}$ with respect to the characteristic cone $\mathcal{C}_{\lambda}$ of (7.1). The equation of this plane is (5.3) where

$$
\begin{aligned}
\rho= & -\lambda^{\prime}+\left(\beta+\gamma \lambda^{3}\right)^{-1}\left[\beta^{2}-\beta(\log \gamma)_{u} \lambda+\left(3 \beta_{v}+\beta(\log \gamma)_{v}\right) \lambda^{2}\right. \\
& \left.-\left(3 \gamma_{u}+\gamma(\log \beta)_{u}\right) \lambda^{4}+\gamma(\log \beta)_{v} \lambda^{5}-\gamma^{2} \lambda^{6}\right] .
\end{aligned}
$$

This plane intersects $\pi_{x}$ in the tangent $t_{\lambda}$. It coincides with the osculating plane of $C_{\lambda}$ if, and only if, the value of $\rho$ in (7.2) is equal to that defined by (5.6). This condition reduces to the differential equation which defines the system of pangeodesics

$$
2\left(\beta+\gamma \lambda^{3}\right) \lambda^{\prime}=\beta_{u} \lambda+2 \beta_{v} \lambda^{2}-2 \gamma_{u} \lambda^{4}-\gamma_{v} \lambda^{5} .
$$

TheOREM 7.1. The plane $\pi_{-\lambda}$ coincides with the osculating plane of $C_{\lambda}$ at $x$ 
if, and only if, the curve $C_{\lambda}$ is a pangeodesic.

The pangeodesic curvature of a curve $C_{\lambda}$ will be defined as the cross ratio of $\pi_{-\lambda}$ with respect to the fundamental set of planes $\pi_{x}, \pi_{0}, \pi_{1}$ (considered in $\S 6$ ), and denoted by $\sigma_{2}(u, v)$. The formula for $\sigma_{2}$ is found to be

$$
\begin{aligned}
\sigma_{2} & =\left(\pi_{x}, \pi_{0}, \pi_{1}, \pi_{-\lambda}\right) \\
& =\left[-2\left(\beta+\gamma \lambda^{3}\right) \lambda^{\prime}+\beta_{u} \lambda+2 \beta_{v} \lambda^{2}-2 \gamma_{u} \lambda^{4}-\gamma_{v} \lambda^{5}\right] /\left(\gamma^{2} \lambda^{6}-\beta^{2}\right) .
\end{aligned}
$$

THEOREM 7.2. The pangeodesic curvature of a curve $C_{\lambda}$ with respect to a quadric (7.1) is independent of the particular choice of the quadric. The pangeodesic curvature of a pangeodesic is equal to zero.

Lane $\left[5\right.$, p. 148] has shown that the asymptotic osculating quadric $Q_{v}$ of Bompiani becomes a quadric of Darboux when $C_{\lambda}$ is tangent to the asymptotic $u$-curve at $x$, and the quadric of Darboux is given by (2.3) where

$$
k_{3}=-\left(\gamma \lambda^{\prime}+\beta \gamma+\theta_{u v}\right) / 2 .
$$

If $C_{\lambda}$ is tangent to an asymptotic curve at $x$, say for example, the $u$-curve, the pangeodesic curvature $\sigma_{2}$ of the curve $C_{\lambda}$ is defined by

$$
\sigma_{2}=2 \lambda^{\prime} / \beta
$$

Then

$$
\lambda^{\prime}=\beta \sigma_{2} / 2
$$

Now if $\lambda^{\prime}$ in formula (7.3) is replaced by its value in (7.4), the following theorem results.

THEOREM 7.3. When $C_{\lambda}$ is tangent to the asymptotic $u$-curve at $x$ and has a constant pangeodesic curvature, the osculating quadric $Q_{v}$ of Bompiani becomes the member of the Darboux pencil (6.3) for which $h=-\sigma_{2} / 2$.

COROLlARY 7.4. If $C_{\lambda}$ is tangent at $x$ to the asymptotic u-curve, the osculating quadratic $Q_{v}$ of Bompiani becomes the quadric of Lie, the quadric of Wilczynski, or the quadric of Fubini, according as the pangeodesic curvature of $C_{\lambda}$ is $0,-2$, or $-2 / 3$, respectively.

The above characterization of the quadric of $\mathrm{Lie}$ is equivalent to the usual definition since the curve $C_{\lambda}$, above defined, becomes the asymptotic $u$-curve in case its pangeodesic curvature is equal to zero.

Similar results to those above can be obtained by using $C_{\lambda}$ in the direction of the asymptotic $v$-curve and the asymptotic osculating quadric $Q_{u}$.

8. The canonical pencil in the canonical plane. Let $l^{\prime}$ denote the line passing through $x$ and the point $z$ whose general coordinates are given by

$$
z=x_{u v}-a x_{u}-b x_{v} \text {. }
$$


The plane (5.3) passes through $l^{\prime}$ if, and only if,

$$
\rho=2 a \lambda^{2}-2 b \lambda \text {. }
$$

The plane $p_{\mu, \lambda}$ with $\mu$ defined by (5.2) contains $l^{\prime}$ when the quadric is chosen such that

$$
k_{3}=-\kappa / 2+\omega,
$$

where

$$
\omega=\beta \gamma\left[(2 a-\psi) \lambda^{2}-(2 b-\phi) \lambda\right] / 2\left(\gamma \lambda^{3}-\beta\right) .
$$

This quadric of Darboux intersects $l^{\prime}$ in the point $P_{1}$ whose general coordinates are given by

$$
z+(a b-\kappa / 2+\omega) x .
$$
by

Let $P_{h}, h=$ const., denote the point whose general coordinates are given

$$
z+(a b-\kappa / 2+h \omega) x .
$$

Therefore, the point $P_{0}$ is the intersection other than $x$ of the quadric of Lie with the line $l^{\prime}$, and the point $P_{\infty}$ is the point $x$. Hence, the point $P_{h}$ is geometrically determined by the cross ratio

$$
\left(P_{\infty}, P_{0}, P_{1}, P_{h}\right)=h .
$$

Let $D_{h}$ denote the quadric of Darboux, which passes through the point $P_{h}$. The equation of $D_{h}$ is (2.3) where $k_{3}$ is given by

$$
k_{3}=h \omega-\kappa / 2 \text {. }
$$

Let $\vartheta_{h}$ denote the polar plane $p_{\mu, \lambda}$ where $\mu$ is given by (5.2) and the quadric used in the definition is the quadric $D_{h}$. The equation of the plane $\vartheta_{h}$ is (5.3) where

$$
\rho=[2 a h+(1-h) \psi] \lambda^{2}-[2 b h+(1-h) \phi] \lambda .
$$

From (8.1) and the form of the coordinates of $P_{h}$ it follows that the correspondence between the points $P_{h}$ and the planes $\vartheta_{h}$ is a projectivity.

As $\lambda$ varies and $h$ is held fixed, the plane $\vartheta_{h}$ generates a pencil whose axis is the line defined by $x^{1}+a^{\prime} x^{3}=0, x^{2}+b^{\prime} x^{3}=0$, where

$$
a^{\prime}=[2 a h+(1-h) \psi] / 2, \quad b^{\prime}=[2 b h+(1-h) \phi] / 2 .
$$

TheOREM 8.1. When $l^{\prime}$ is the canonical line $c^{\prime}(k)$, for which $a=-k \psi$, $b=-k \phi$, the axis of the pencil of planes $\vartheta_{h}$ is the canonical line $c^{\prime}\left(k^{\prime}\right)$ where $k^{\prime}=(1-h-2 k h) / 2$. If $l^{\prime}$ is the projective normal, $k=0$ and $k^{\prime}=(1-h) / 2$.

When $l^{\prime}$ is the $R_{\lambda}^{\prime}$-associate $\left[1\right.$, p. 391] of the canonical line $c^{\prime}(k), a=-k \psi$ 
$-\gamma \lambda$ and $b=-k \phi-\beta / \lambda$, the equation of the plane $\vartheta_{h}$ is (5.3) where

$$
\rho=2 h \beta+(2 k h+h-1) \phi \lambda-(2 k h+h-1) \psi \lambda^{2}-2 h \gamma \lambda^{3} .
$$

TheOREM 8.2. The plane $\vartheta_{h}$ with $\rho$ defined by (8.2) coincides with the osculatlating plane of the curve $C_{\lambda}$ if, and only if, $C_{\lambda}$ is a curve in the system of hypergeodesics defined by

$$
\begin{aligned}
\lambda^{\prime}= & (2 h-1) \beta+\left[\theta_{u}+(2 k h+h-1) \phi\right] \lambda \\
& -\left[\theta_{v}+(2 k h+h-1) \psi\right] \lambda^{2}-(2 h-1) \gamma \lambda^{3} .
\end{aligned}
$$

The cusp-axis of this system is the canonical line which is the axis of the pencil of planes $\vartheta_{h}$ with $\rho$ given by (8.1).

The above constructions based on any given canonical line $c^{\prime}(k)$ yield any desired canonical line $c^{\prime}\left(k^{\prime}\right)$ in the canonical plane for a suitable choice of $D_{h}$. The constructions for $h=0$ and $h=\infty$ yield, independently of $k$, the directrix $d^{\prime}$ of Wilczynski and the canonical tangent $t^{\prime}$, respectively.

9. The $K$-associate quadrics. Quadrics having contact of second order with $S$ at $x$ exist which are such that their characteristic cones for an arbitrary direction $\lambda$ are tangent to $\pi_{x}$. There exists a two-parameter family of those quadrics which are characterized by the additional property that at every point $x$ of $S$ the direction $\mu$ of the line of contact of the characteristic cones of the family with $\pi_{x}$ makes a constant cross ratio with the fundamental set which consists of the asymptotic directions and the direction $\lambda$ at $x$. That is, $(\infty, 0, \lambda, \mu)=K, K=$ const. From this cross ratio equation it is clear that

$$
\mu=K \lambda \text {. }
$$

The quadrics thus defined will be called the $K$-associate quadrics of $S$ at $x$ and such a quadric will be denoted by $Q_{K}$. A characteristic cone of a quadric $Q_{K}$ with respect to the curve $C_{\lambda}$ will be denoted by $\mathcal{C}_{\lambda, K}$.

To verify the above assertion and to obtain the equation of the $K$-associate quadrics of $S$ note from equations (3.7) that the lines of intersection of $C_{\lambda}$ with $\pi_{x}$ coincide if, and only if,

$$
\left(k_{1}+\lambda k_{2}\right)^{2}=\left(\gamma \lambda+k_{2}\right)\left(\beta+\lambda k_{1}\right) .
$$

The direction of the line of contact is given by

$$
x^{2} / x^{1}=-\left(k_{1}+\lambda k_{2}\right) /\left(\gamma \lambda+k_{2}\right) .
$$

Demanding that this direction coincide with $K \lambda$ and solving (9.1) and (9.2) for $k_{1}$ and $k_{2}$ yields

$$
\begin{aligned}
& k_{1}=\left[K^{2} \gamma \lambda^{3}-(K+1) \beta\right] /\left(K^{2}+K+1\right) \lambda, \\
& k_{2}=\left[\beta-\left(K^{2}+K\right) \gamma \lambda^{3}\right] /\left(K^{2}+K+1\right) \lambda^{2} .
\end{aligned}
$$

Since equation (2.2) subject to conditions (9.3) involves two arbitrary parameters $K, k_{2}$, the $K$-associate quadrics form a two-parameter family. 
The quadrics of the Moutard pencil and the quadrics of Davis for a direction $\lambda$ form the one-parameter families of $K$-associate quadrics for which $K=1$, and $K=-1$, respectively. The one-parameter families of $K$-associate quadrics for which $K=0$ and $K=\infty$ contain the asymptotic osculating quadrics $Q_{u}$ and $Q_{v}$ of Bompiani for the curve $C_{\lambda}$, respectively. It seems appropriate, therefore, to call these families for which $K=0$ and $K=\infty$ the families of asymptotic associate quadrics.

10. Transformation of Čech and conjugate directions. The transformation $\Sigma_{h}$ of Cech is a one-to-one correspondence between an arbitrary point $P$ in $\pi_{x}$ and a plane $\pi$ which passes through the point $x$. The purpose of this section is to present new geometric characterizations of this transformation and of conjugate directions.

Let $y^{0}, y^{1}, y^{2}, 0$ and $0, \xi_{1}, \xi_{2}, \xi_{3}$ denote local homogeneous coordinates of the point $P$ and plane $\pi$ with respect to the reference tetrahedron whose vertices are $x, x_{u}, x_{v}, x_{u v}$. The point $P$ and plane $\pi$ correspond by the transformation $\Sigma_{h}$ of Cech if, and only if, their coordinates are related by the equations

$$
\begin{aligned}
\xi_{0} & =0, \quad \sigma \xi_{1}=y^{1}\left(y^{2}\right)^{2}, \quad \sigma \xi_{2}=\left(y^{1}\right)^{2} y^{2}, \\
\sigma \xi_{3} & =-y^{0} y^{1} y^{2}+h\left[\beta\left(y^{1}\right)^{3}+\gamma\left(y^{2}\right)^{3}\right] .
\end{aligned}
$$

The equation of the polar plane of $P$ with respect to the quadric $Q_{K}$ is

$$
y^{2} x^{1}+y^{1} x^{2}+\left(k_{1} y^{1}+k_{2} y^{2}-y^{0}\right) x^{3}=0 .
$$

The local plane coordinates of this plane are therefore given by

$$
\xi_{0}=0, \quad \sigma \xi_{1}=y^{2}, \quad \sigma \xi_{2}=y^{1}, \quad \sigma \xi_{3}=k_{1} y^{1}+k_{2} y^{2}-y^{0},
$$

where $k_{1}, k_{2}$ are defined by (9.3). These equations may be written in the form

$$
\xi_{0}=0, \quad \sigma \xi_{1}=\lambda y^{1}, \quad \sigma \xi_{2}=y^{1}, \quad \sigma \xi_{3}=\left(k_{1}+k_{2} \lambda\right) y^{1}-y^{0},
$$

where $y^{2}$ is replaced by $\lambda y^{1}$. The local coordinates of the plane $\pi$ which corresponds to a point $P$ on $t_{\lambda}$ are found to be

$$
\begin{aligned}
\xi_{0} & =0, \quad \sigma \xi_{1}=\lambda^{2}\left(y^{1}\right)^{3}, \quad \sigma \xi_{2}=\lambda\left(y^{1}\right)^{3}, \\
\sigma \xi_{3} & =-\lambda y^{0}\left(y^{1}\right)^{2}+h\left(\beta+\gamma \lambda^{3}\right)\left(y^{1}\right)^{3} .
\end{aligned}
$$

Multiplying equations $(10.1)$ by $\left(y^{1}\right)^{2}$ and replacing $k_{1}$ and $k_{2}$ by their values from (9.3) reveals that the polar plane (10.1) and the plane $\pi$ coincide if, and only if, $h$ and $K$ are related by the equation

$$
h K^{2}+(h+1) K+h=0 \text {. }
$$

The roots of this equation are reciprocals.

THEOREM 10.1 The polar plane of an arbitrary point $P$ in $\pi_{x}$ with respect to the quadric $Q_{K}$ determined for the direction of the line joining $x$ and $P$ is the plane 
which corresponds to $P$ in the transformation $\Sigma_{h}$ of $\nearrow_{\text {ech, }}$ where $h$ and $K$ are related by (10.2). There are two families of quadrics $Q_{K}$ and $Q_{K}{ }^{-1}$ which serve to characterize geometrically each particular transformation $\Sigma_{h}$.

A general pair of quadrics $Q_{K}, Q_{K}^{-1}$ will be called Čech associate quadrics. These quadrics become self-associates when $K= \pm 1$. Equation (10.2) has double roots -1 if $h=1$ and double roots +1 if $h=-1 / 3$.

Specializations of Theorem 10.1 yield geometric characterizations for the correspondence of Segre $\Sigma_{1}$, the correspondence of Moutard [6, p. 508] $\Sigma_{-1 / 3}$, the polarity of Lie $\Sigma_{0}$, and the associate correspondence of Segre [4, p. 233] $\Sigma_{-1}$. The correspondence of Segre, the correspondence of Moutard, and the polarity of Lie are induced by the Cech self-associate quadrics $Q_{-1}$ (the quadrics of Davis), the Cech self-associate quadrics $Q_{1}$ (the quadrics of the Moutard pencil), and the asymptotic associate quadrics, respectively.

Let $\mu=K \lambda, \mu_{i}=K_{i} \lambda, i=1,2,3$, where $K_{1}, K_{2}$ are the roots of (10.2), and $K_{3}=1$. The cross ratio equation

$$
\left(\mu_{1}, \mu_{2}, \mu_{3}, \mu\right)=-1
$$

is satisfied if, and only if, $K=-1$. The following new geometric characterization of conjugate directions may now be stated.

THEOREM 10.2. The lines of contact of an arbitrary pair of cones $\mathcal{C}_{\lambda, K}$ and $\mathcal{C}_{\lambda, K^{-1}}$ with the plane $\pi_{x}$ separate harmonically the conjugate tangents $t_{\lambda}$ and $t_{-\lambda}$.

11. Pangeodesics. Moutard quadric. Asymptotic osculating quadrics. The equation of the cone $C_{\lambda, K}$ is found by replacing $k_{1}$ and $k_{2}$ in equation (3.4) by their values in (9.3) to be

$$
\begin{aligned}
K^{2}\left(x^{1}\right)^{2}-2 K \lambda^{-1} x^{1} x^{2}+\lambda^{-2}\left(x^{2}\right)^{2} & +2 \rho a_{13} x^{1} x^{3} \\
& +2 \rho a_{23} x^{2} x^{3}+2 \rho a_{33}\left(x^{3}\right)^{2}=0,
\end{aligned}
$$

where $\rho=\left(K^{2}+K+1\right) / 2\left(\beta+\gamma \lambda^{3}\right)$ and $a_{13}, a_{23}, a_{33}$ are the coefficients of $x^{1} x^{3}$, $x^{2} x^{3},\left(x^{3}\right)^{2}$, respectively, in equation (3.4) af ter $k_{1}$ and $k_{2}$ have been replaced by their values in (9.3). The necessary and sufficient condition that $\mathcal{C}_{\lambda, \boldsymbol{K}}$ reduce to two planes is that the discriminant of (11.1) be equal to zero. This condition is found to be

$$
\begin{aligned}
(K+1) k_{3}= & \lambda^{-2} \alpha(1-K)\left(\beta+K^{2} \gamma \lambda^{3}\right) \lambda^{\prime} \\
& -2 \lambda^{-1} \alpha(K+1)^{2}\left[\beta^{2}+\left(K^{3}+1\right) \beta \gamma \lambda^{3}+K^{3} \gamma^{2} \lambda^{6}\right] \\
& -2^{-1}(K+1) \theta_{u v}+\alpha\left[K \beta_{u} \lambda^{-1}-\left(2 K^{2}+3 K+3\right) \beta_{v}\right. \\
& \left.-\left(3 K^{3}+3 K^{2}+2 K\right) \gamma_{u} \lambda^{2}+K^{2} \gamma_{v} \lambda^{3}\right] \\
& -\alpha(K+1)\left[-\beta(\log \gamma)_{u} \lambda^{-1}+(K+1) \beta(\log \gamma)_{v}\right. \\
& \left.+K(K+1) \gamma(\log \beta)_{u} \lambda^{2}-K^{2} \gamma(\log \beta)_{v} \lambda^{3}\right],
\end{aligned}
$$

where $\alpha=\left[2 \lambda\left(K^{2}+K+1\right)\right]^{-1}$. 
TheOrem 11.1. The cone $C_{\lambda, K}$ reduces to two planes if, and only if, $k_{3}$ is defined by the formula (11.2). These two planes intersect in a line of $\pi_{x}$ whose direction is $K \lambda$.

The following geometric characterizations of the pangeodesics, the Moutard quadric, and asymptotic osculating quadrics can be deduced by substituting proper values of $K$ in formula (11.2).

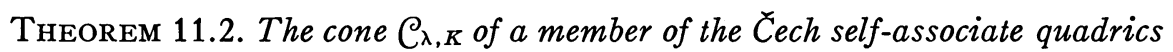
$Q_{-1}$ reduces to two planes if, and only if, the curve $C_{\lambda}$ is a pangeodesic.

THEOREM 11.3. The cone $\mathcal{C}_{\lambda, K}$ of a member of the Cech self-associate quadrics $Q_{1}$ reduces to two planes if, and only if, $Q_{1}$ is the Moutard quadric for the direction $\lambda$. These two planes, $\pi_{x}$, and the osculating plane of $C_{\lambda}$ at $x$ form a harmonic set.

The proof of the second part of the above theorem is left to the reader.

THEOREM 11.4. The cone $\mathcal{C}_{\lambda, K}$ of a member of the asymptotic associate quadrics $Q_{0}\left(Q_{\infty}\right)$ reduces to two planes if, and only if, $Q_{0}\left(Q_{\infty}\right)$ is the asymptotic osculating quadric $Q_{u}\left(Q_{v}\right)$ of Bompiani for the curve $C_{\lambda}$ at $x$.

12. The class $\mathfrak{B}$ of generalized pangeodesics. The equation of the polar plane of any point $P$ on an arbitrary tangent $t_{\mu}$ with respect to the cone $\mathcal{C}_{\lambda, K}$ is found by substituting the values for $k_{1}$ and $k_{2}$, given by (9.3), in equation (3.5) to be

$$
2 K \lambda^{2} x^{1}-2 \lambda x^{2}+L x^{3}=0
$$

where $L$ is defined by

$$
\begin{aligned}
L= & \left\{\left[-(K+1) \beta \lambda+2 \beta \mu-2 K^{2} \gamma \lambda^{4}+\left(K^{2}+K\right) \gamma \lambda^{3} \mu\right] \lambda^{\prime}\right. \\
& +\left(K^{2}+K+1\right)^{-1}\left[\left(2 K^{2}+2 K+1\right) \beta^{2} \lambda-K \mu \beta^{2}\right. \\
& +\left(-K^{3}+2 K^{2}+2 K+1\right) \beta \gamma \lambda^{4}+\left(K^{4}+2 K^{3}+2 K^{2}-K\right) \beta \gamma \mu \\
& \left.-K^{3} \gamma^{2} \lambda^{7}+\left(K^{4}+2 K^{3}+2 K^{2}\right) \gamma^{2} \lambda^{6} \mu\right]-\beta_{u} \lambda \mu \\
& +\left(2 K^{2}+3 K+3\right) \beta_{v} \lambda^{3}+\left(3 K^{2}+3 K+2\right) \gamma_{u} \lambda^{4} \mu-K^{2} \gamma_{v} \lambda^{6} \\
& +K^{2} \gamma(\log \beta)_{u} \lambda^{5}+\left(K^{2}+K+1\right) \gamma(\log \beta)_{u} \lambda^{4} \mu-(K+1) \beta(\log \gamma)_{u} \lambda^{2} \\
& -\left(K^{2}+K\right) \gamma(\log \beta)_{v} \lambda^{5} \mu+\left(K^{2}+K+1\right) \beta(\log \gamma)_{v} \lambda^{3}+\beta(\log \gamma)_{v} \lambda^{2} \mu \\
& \left.+\left(K^{2}+K+1\right)(\lambda+\mu)\left(\theta_{u v}+2 k_{3}\right)\right\} /(K \lambda-\mu)\left(\beta+\gamma \lambda^{3}\right) .
\end{aligned}
$$

The polar plane of an arbitrary point on $t_{\mu}$ with respect to the cone $\mathcal{C}_{\lambda, K}$ will be designated by $q_{\mu}$. The polar plane $q_{\mu}$ intersects $\pi_{x}$ in the line whose direction is $K \lambda$. The equation of the osculating plane of the curve $C_{K \lambda}$ at $x$ is given by (12.1) where

$$
L=\lambda_{u}+K \lambda \lambda_{v}+\beta / K-\theta_{u} \lambda+K \theta_{v} \lambda^{2}-K^{2} \gamma \lambda^{3} .
$$

The polar plane $q_{\mu}$, therefore, coincides with the osculating plane of $C_{K \lambda}$ at $x$ 
if, and only if, the values of $L$ defined by (12.2) and (12.3) are equal. When $K=-1$, this condition is independent of the choice of $\mu$ and the following theorem can be stated.

THEOREM 12.1. The polar plane of any point $P$ in the tangent plane of $S$ at $x$ with respect to a cone $C_{\lambda, K}$ of a member of the Cech self-associate quadrics $Q_{-1}$ coincides with the osculating plane of $C_{K \lambda}$ if, and only if, $C_{\lambda}$ is a pangeodesic.

In this case in which $C_{\lambda}$ is a pangeodesic the cone $C_{\lambda, K}$ reduces to two planes (Theorem 11.2) which separate harmonically the plane $\pi_{x}$ and the polar plane of an arbitrary point of $\pi_{x}$ with respect to the planes of $C_{\lambda, K}$.

In case $\mu$ is the conjugate direction $-\lambda$ of $\lambda$, the equation formed by equating the values of $L$ given by (12.2) and (12.3) reduces to

$$
\begin{aligned}
& {\left[2(K+2) \beta+\left(3 K^{2}+2 K+1\right) \gamma \lambda^{3}\right] \lambda_{u}+\left[\left(K^{2}+2 K+3\right) \beta\right.} \\
& \left.\quad+\left(4 K^{2}+2 K\right) \gamma \lambda^{3}\right] \lambda \lambda_{v}=(K+2) \beta_{u} \lambda+\left(K^{2}+2 K+3\right) \beta_{v} \lambda^{2} \\
& \quad-\left(3 K^{2}+2 K+1\right) \gamma_{u} \lambda^{4}-\left(2 K^{2}+K\right) \gamma_{v} \lambda^{5} \\
& \quad+K^{-1}(K-1)(K+1)^{2}\left(K^{2}+K+1\right)^{-1}\left(\beta+\gamma \lambda^{3}\right)\left(\beta+K^{3} \gamma \lambda^{3}\right) .
\end{aligned}
$$

Definition: A system of curves each curve $C_{\lambda}$ of which is characterized by the property that as $x$ moves along $C_{\lambda}$ the polar plane $q_{-\lambda}$ coincides with the osculating plane of $C_{K \lambda}$ at $x$ will be called a system $\mathfrak{B}_{K}$ of the class $\mathfrak{B}$ of generalized pangeodesics of $S$ at $x$. The differential equation of the system $\mathfrak{P}_{K}$ is (12.4).

Since (12.4) reduces to the equation for the pangeodesics when $K= \pm 1$, the following theorem results.

TheOREM 12.2. The systems $\mathfrak{B}_{1}$ and $\mathfrak{B}_{-1}$ of curves of the class $\mathfrak{B}$ of generalized pangeodesics coincide in the system of pangeodesics of $S$ at $x$.

Since (12.4) reduces to the equation of the curves of Darboux for $K=0$ and $K=\infty$, the following theorem results.

THEOREM 12.3. The curves of the systems $\mathfrak{P}_{0}$ and $\mathfrak{B}_{\infty}$ of the class $\mathfrak{B}$ of generalized pangeodesics coincide. The curves, thus characterized, are the curves of Darboux.

It is noteworthy that the quadrics employed in the above characterizations of the pangeodesics and the curves of Darboux are the Cech self-associate quadrics and the asymptotic associate quadrics, respectively.

The following theorem may be verified by the reader.

THEOREM 12.4. The polar plane of an arbitrary point on $t_{\lambda}$ with respect to the characteristic cone $\mathcal{C}_{\lambda, K}$ of the member of the Čech self-associate quadrics $Q_{-1}$ for which $k_{3}=\theta_{u v} / 2$ coincides with the osculating plane of $C_{-\lambda}$ if, and only if, $C_{\lambda *}$ is a hypergeodesic defined by 


$$
\lambda^{* \prime}=\left[2 \theta_{u}+(\log \gamma)_{u}\right] \lambda^{*}-\left[2 \theta_{v}+(\log \beta)_{v}\right] \lambda^{* 2}
$$

where $\lambda^{*}$ is the $N_{\lambda}$-correspondent $[3$, p. 538] of $\lambda$ defined by

$$
\lambda^{*}=-\gamma \lambda^{4} / \beta \text {. }
$$

The cusp-axis of these hypergeodesics is the canonical line $c^{\prime}(-1 / 2)$. This cuspaxis and the directrix $d^{\prime}$ of Wilczynski separate harmonically the projective normal $n^{\prime}$ of Fubini and the canonical tangent $t^{\prime}$.

\section{REFERENCES}

1. P. O. Bell, A study of curved surfaces by means of certain associated ruled surfaces, Trans. Amer. Math. Soc. vol. 46 (1939) pp. 389-409.

2. - A study of the projective differential geometry of surfaces by means of a modified tensor analysis, Trans. Amer. Math. Soc. vol. 60 (1946) pp. 22-50.

3. - On differential geometry intrinsically connected with a surface element of projective arc length, Trans. Amer. Math. Soc. vol. 50 (1941) pp. 529-547. 234.

4. V. G. Grove, The transformation of Cech, Bull. Amer. Math. Soc. vol. 50 (1944) pp. 231-

5. E. P. Lane, $A$ treatise on projective differential geometry, University of Chicago Press, 1942.

6. G. Wu, Systems of quadrics associated with a point on a surface, I, Duke Math. J. vol. 10 (1943) pp. 499-513.

Kansas State College,

Manhattan, Kan.

The University of Kansas,

LAWRENCE, KAN. 